\title{
Silvicultura de los bosques de Fitzroya cupressoides. ¿Ficción o realidad?
}

\author{
Silviculture of the Fitzroya cupressoides forest. Fiction or reality? \\ C.D.O. 568 \\ CLAUDIO DONOSO ${ }^{1}$, VICTOR SANDOVAL ${ }^{2}$ y RENATO GREZ
${ }^{1}$ Instituto de Silvicultura; ${ }^{2}$ Institute de Manejo y Economía Forestal,
Universidad Austral de Chile, Casilla 567 , Valdivia, Chile
}

\begin{abstract}
SUMMARY
Fitzroya cupressoides is still an important part of forest surfaces and timber volumes, having been exploited with only mining criteria for very long. The possibility of applying silvicultural treatments in the Andean and Coastal Cordillera Alerce forests was analyzed using the knowledge acquired and having in mind a timber production objective with a sustained yield criteria that would be in harmony with the essential forest values. The results indicate that it is possible, from a silvicultural point of view, to exploit the Alerce forest of Contao.

However, the forest values related to a millenarian and unique substrate, as well as the presence of very old fossil soils indicate that to maintain the population structure, only silvicultural actions should be applied.
\end{abstract}

\section{RESUMEN}

Fitzroya cupressoides constituye aún importantes superficies de bosques y volúmenes de madera que, desde muy antiguo, han sido utilizados sólo con criterios extractivos de tipo minero. Sobre la base de los conocimientos acumulados sobre los bosques de alerce en los últimos años y teniendo presente un objetivo de producción de madera con un criterio de rendimiento sostenido y de mantención de los valores esenciales del bosque, se intenta en esta contribución analizar la posibilidad de aplicación de tratamientos silviculturales en bosques de la Cordillera de los Andes y de la Costa. Se concluye que existe la posibilidad técnica silvicultural de explotar el bosque de alerce de Contao, pero que los valores relacionados no sólo con el dosel arbóreo, sino que con aquellos representados por un sustrato milenario y único, con presencia de suelos fósiles muy antiguos, indican que, salvo acciones silviculturales puramente destinadas a mantener la estructura poblacional, toda el área debería ser traspasada al Sistema Nacional de Areas Protegidas y, más aún, se propone la creación de una Reserva de la Biósfera. En el caso de los bosques de la Cordillera de la Costa de Valdivia, en el sector de Venecia, se concluye que la aplicación de un método de cortas de selección uniforme es no sólo factible de llevarse a la práctica, sino que se estima que constituye una necesidad para ordenar silviculturalmente los bosques, regenerar las áreas alteradas y lograr que el ecosistema retorne a su equilibrio natural.

\section{INTRODUCCION}

De acuerdo con la legislación vigente (D.F.L. 4 de 1976), Fitzroya cupressoides (Mol.) Johnston es una especie que no puede cortarse ni comercializarse en estado verde. Esa legislación, lo mismo que las disposiciones internacionales que prohiben la comercialización de la madera de alerce a los países miembros de CITES (Convención Internacional para el Comercio de Especies de Flora y Fauna Amenazada), tiene por objeto proteger de una posible extinción a una especie que posee relevantes valores desde el punto de vista científico, cultural y estético. Considerando las restricciones impuestas por las leyes podría pensarse que tiene poco sentido discutir respecto de la silvicultura de bosques de tales características, como las del Tipo Forestal Alerce. Sin embargo, a los autores de este trabajo no les ha parecido así. La historia del alerce, como ya se ha señalado en numerosos trabajos (Veblen et al., 1976; Veblen y Ashton, 1982; Donoso et al., en prensa; Parker y Donoso*), no es otra cosa que una larga lista de explotaciones que significaron grandes cantidades de dinero para algunos pocos propietarios, pero especialmente enormes superficies devastadas por talas rasas indiscriminadas y por incendios de gigantescas dimensiones, como aque-

* Comunicación personal. 
llos relatados por Pérez Rosales (1850) o Elizalde (1971). En las localidades, provincias o regiones, desde donde se extrajo el recurso, no quedó nada, aparte de los bosques quemados o alterados drásticamente; no quedaron pueblos ni villorrios, no quedaron caminos transitables, no quedaron puentes $\mathrm{y}$, en muchos sitios, no quedaron tampoco bosques jóvenes, ni siquiera de otras especies distintas de $F$. cupressoides, que por lo menos permitieran vislumbrar un futuro mejor.

Es indudable que la aplicación de criterios silviculturales y sociales un poco más evolucionados habría significado bosques, caminos, pueblos distintos de los existentes hoy. Desde luego, no habría un decreto nacional ni disposiciones internacionales que impidieran la utilización de la madera de $F$. cupressoides; esta especie no estaría en la lista de especies amenazadas y habría bosques más ordenados que permitirían la aplicación de una silvicultura moderna sin gran complicación.

Los grandes bosques de alerce que aún permanecen en la región de Contao, así como las evidencias que indican de qué dimensiones comparables fueron los de la Cordillera de la Costa (Donoso et al., en prensa) y los que había en las superficies hoy cubiertas de tocones del Llano Central, al norte de Puerto Montt (Parker y Donoso*), dan testimonio de que una silvicultura adecuada a las especies, a las superficies y a los volúmenes existentes era evidentemente posible. Pero, acaso, ¿no es posible aún?; es indudable que una respuesta clara a esta pregunta la puede dar solamente un estudio del recurso actual y un análisis de las posibilidades técnicas de aplicar silvicultura en él, y que debe incluir, naturalmente, las posibilidades de regeneración, de volteo, de madereo, transporte y los costos correspondientes. Sólo entonces podremos decidir con certeza si la silvicultura en el Tipo Forestal Alerce es realidad o sólo ficción.

Por ahora creemos que se ha adquirido un conocimiento suficiente del recurso sobre la base de los estudios realizados en los últimos años (Garrido et al., 1983; Donoso et al., 1987; Donoso et al., en prensa). En la actualidad se realizan, bajo el auspicio de CONAF, los estudios experimentales tendientes a verificar las posibilidades de corta, de regeneración y de madereo de la especie en los bosques de la Cordillera de la Costa de Valdivia. La aplicación de esta experiencia se está realizando tomando como base el análisis de los métodos silviculturales posibles de aplicar a los subtipos del Tipo Forestal Alerce determinados por Donoso et al. (1987). El desarrollo de este análisis es el propósito del presente trabajo.

* Comunicación personal.

\section{METODOLOGIA}

Para realizar el análisis silvicultural del Tipo Forestal Alerce, como de cualquier otra clase de bosques, es necesario definir primero el objetivo que se persigue. En este caso se trata de lograr el máximo de producción de madera aserrable de Fitzroya cupressoides en forma sostenida en el tiempo, y en armonía con los valores de vida silvestre, recursos hídricos, belleza del paisaje y recreación.

Con ese objetivo en mente se acudió al estudio de los ecosistemas del tipo forestal, lo que se realizó en dos áreas definidas, una representativa de la Cordillera de los Andes, en la región de Contao, de aproximadamente 25.000 hectáreas, y la otra, en la Cordillera de la Costa de Valdivia, de aproximadamente 15.000 hectáreas. En estos ecosistemas se efectuó un análisis de las características del medio ambiente físico, de las especies forestales principales, en especial $F$. cupressoides, y de las características de composición, estructura y dinámica de los bosques. Las características del medio ambiente consideradas fueron: clima, suelo, geomorfología y topografía; ellas fueron evaluadas y analizadas mediante la información existente de estaciones meteorológicas próximas, de la literatura existente en cuanto a geomorfología y suelos, de la observación y toma de datos en terreno en cuanto a suelos y topografía y de la interpretación de estos datos para efectos de microclima y micrositio en general. Las características ecológicas de $F$. cupressoides, así como las estructuras de los bosques, fueron analizadas tomando como base alguna literatura existente sobre germinación, semillación, requerimientos de sitio y edáficos, regeneración natural y dinámica regenerativa, así como toma de datos y su análisis correspondiente para obtener información sobre composición y estructura de los bosques. Esta información y el análisis correspondiente fueron volcados en un documento de 4 tomos (Donoso et al., 1987) y en tres manuscritos previos a éste (Donoso, en prensa; Donoso et al., en prensa).

Las características del medio ambiente físico fueron utilizadas en el análisis de métodos silviculturales, como un antecedente básico que permite, restringe o impide la aplicación de determinado método de corta y regeneración o tipo de intervención en el bosque. Las características del bosque y de las especies constituyentes, en especial $F$. cupressoides, fueron utilizadas en el análisis como otro elemento básico, según el cual la aplicación de determinado método silvicultural es posible con mayor o menor dificultad o, en otras palabras, el elemento básico que indica el método posible de aplicar para lograr la composición y 
estructura del bosque que se desea en función del objetivo planteado.

Para decidir la posibilidad de aplicar un método silvicultural que sea compatible con las restricciones impuestas por el medio ambiente físico, así como con las características ecológicas de las especies y el tipo de estructura y composición deseado, es básico contar con los antecedentes de superficies, volúmenes y crecimiento de los distintos tipos de bosques o subtipos forestales previamente definidos.

Los métodos de corta y regeneración discutidos a la luz de los antecedentes de los ecosistemas de $F$. cupressoides son todos aquellos señalados con sus modificaciones por Hawley y Smith (1972) y por Daniel et al. (1982). Un análisis general de los métodos enfrentados primero a las restricciones impuestas por el medio ambiente, por el bosque y por las especies, y luego, según el resultado de ello, a las posibilidades otorgadas por las superficies, volúmenes y crecimientos disponibles, permitirá determinar, en primera aproximación, la factibilidad técnica, desde el punto de vista puramente silvícola-ecológico, de aplicar determinados métodos.

\section{RESULTADOS Y DISCUSION}

\section{SUBTIPOS DE ALERCE ANDINO}

Métodos de corta de tala rasa y árbol semillero. Del estudio planimétrico de los planos tipológicos se obtuvo la información de superficies de los subtipos de alerce andino, según las categorías de pendiente definidas de acuerdo con la legislación (Regl. N N $^{\circ} 59$ del D.L. 701 de 1974) (cuadro 1).
La observación del cuadro 1 permite verificar que en casi un $24 \%$ de la superficie total del tipo forestal en Contao, la pendiente es superior al $60 \%$, pendiente extremadamente escarpada que por sí sola descarta cualquier tipo de intervención sobre ella. Un $15.5 \%$ de la superficie total posee pendientes superiores a $45 \%$, que se consideran como muy escarpadas y que, dadas las condiciones de precipitación muy alta, profundidad y drenaje del suelo, tipo de sustrato subyacente y ocurrencia de fenómenos de tixotropía (Kuhne, 1985), permiten predecir problemas de solifluxión y escurrimiento de suelos. Es decir, la superficie con esta categoría de pendiente también debe considerarse excluida de intervención silvícola.

De acuerdo con lo señalado queda del orden de un $60 \%$ de la superficie de bosques con pendientes inferiores al $45 \%$, respecto de la cual podría discutirse la posibilidad de incorporarla a un sistema de utilización mediante técnicas silvícolas que deberían tener la condición de mantener o mejorar la potencialidad del Tipo Forestal Alerce.

Para aplicar un método silvicultural en la superficie de bosques que queda es necesario conocer, además de las superficies, los volúmenes por categorías de pendientes y las tasas de crecimiento de los bosques que interesan. La estimación de los volúmenes por categoría de pendiente, obtenida del inventario general (cuadro 2), permite determinar, para pendientes de 0 a $45 \%$, un volumen aserrable de 62.846 .140 pulgadas madereras del tipo Alerce-Coigüe de Chiloé; de 5.364 .065 p.m. del subtipo Alerce-Coigüe de Magallanes, y de 2.395.886 p.m. del subtipo Alerce-Ciprés de las Guaitecas.

Sin embargo, parte de las superficies correspondientes a esas categorías de pendientes debe excluirse de una eventual utilización maderera del

\section{CUADRO 1}

Superficie (há) de subtipos del Tipo Forestal Alerce de la Cordillera de los Andes (Contao), según categoría de pendiente.

Area (ha) per slopes classes of the subtypes of the Alerce Forest Type of the Andean Cordillera (Contao).

\begin{tabular}{|c|c|c|c|c|c|c|}
\hline \multirow{2}{*}{ Subtipo forestal } & \multicolumn{4}{|c|}{ Categorías de pendientes } & \multirow{2}{*}{ Total } & \multirow{2}{*}{$\%$} \\
\hline & $0-30 \%$ & $31-45 \%$ & $46-60 \%$ & $60 \%$ y más & & \\
\hline Alerce-Coigüe, Chiloé & 4.589 & 3.501 & 2.371 & 3.487 & 13.948 & 56.9 \\
\hline Alerce-Coigüe, Magallanes & 1.622 & 1.060 & 1.116 & 1.785 & 5.579 & 23.0 \\
\hline Alerce-Ciprés & 582 & 24 & & 20 & 627 & 2.5 \\
\hline Alerce marginal & 721 & 240 & $20 \overline{8}$ & 432 & 1.601 & 6.5 \\
\hline Alerce explotado & 1.810 & 716 & 90 & 110 & 1.726 & 11.1 \\
\hline Totales & $\begin{array}{c}9.324 \text { há } \\
38.1 \%\end{array}$ & $\begin{array}{c}5.541 \text { há } \\
22.6 \%\end{array}$ & $\begin{array}{c}3.785 \text { há } \\
15.5 \%\end{array}$ & $\begin{array}{l}5.834 \text { há } \\
23.8 \%\end{array}$ & $\begin{array}{c}23.481 \text { há } \\
100 \%\end{array}$ & 100 \\
\hline
\end{tabular}




\section{CUADRO 2}

Volúmenes de Fitzroya cupressoides de los subtipos del Tipo Forestal Alerce Andino en las diferentes categorías de pendiente en el sector de Contao.

Fitzroya cupressoides timber volumes of the subtypes of the Andean Alerce Forest Type in the different slope classes in Contao.

\begin{tabular}{|c|c|c|c|c|c|}
\hline \multirow[b]{2}{*}{ Subtipo forestal } & \multicolumn{4}{|c|}{ Categorías de pendientes } & \multirow{2}{*}{ Total } \\
\hline & $0-30 \%$ & $31-45 \%$ & $46-60 \%$ & $60 \%$ y más & \\
\hline \multicolumn{6}{|c|}{ Volumen (miles de $\mathrm{m}^{3}$ s.s.c.) } \\
\hline Alerce-Coigüe, Chiloé & 3.000 & 2.284 & 1.544 & 2.301 & 9.124 \\
\hline Alerce-Coigüe, Magallanes & 321 & 210 & 221 & 354 & 1.106 \\
\hline Alerce-Ciprés & 175 & 7 & - & 6 & 188 \\
\hline Alerce marginal & 47 & 16 & 14 & 28 & 105 \\
\hline Total & 3.543 & 2.517 & 1.779 & 2.689 & 10.528 \\
\hline \multicolumn{6}{|c|}{ Volumen (miles pulgadas madereras) } \\
\hline Alerce-Coigüe, Chiloé & 35.711 & 27.136 & 18.344 & 27.353 & 108.544 \\
\hline Alerce-Coigüe, Magallanes & 3.241 & 2.123 & 2.235 & 3.576 & 11.175 \\
\hline Alerce-Ciprés & 2.302 & 94 & - & 79 & 2.475 \\
\hline Alerce marginal & 483 & 161 & 139 & 210 & 1.073 \\
\hline Total & 41.737 & 29.514 & 20.718 & 31.298 & 123.267 \\
\hline
\end{tabular}

recurso, dentro del marco de una sana política forestal frente a bosques de estas características. De las 8.090 hectáreas del subtipo Alerce-Coigüe de Chiloé, en el rango de pendientes inferiores a $45 \%$, deben excluirse por lo menos 2.000 há, que corresponderían a situaciones de protección de laderas y cuencas, y, principalmente, que deberían anexarse al contiguo Parque Nacional Hornopirén, por el hecho de no encontrarse ninguna área de este tipo de bosque bajo el resguardo del Sistema Nacional de Areas Silvestres de Protección. De las 2.788 há del subtipo Alerce-Coigüe de Magallanes deberían excluirse por lo menos 1.000 há, que se estima deben encontrarse en áreas de protección de cuencas y laderas. Esto daría, en forma aproximada, en hectáreas aprovechables: 6.000 para el subtipo Alerce-Coigüe de Chiloé; 1.500 para Alerce-Coigüe de Magallanes, y 600 para Alerce-Ciprés.

De los cuadros 1 y 2 se puede desprender que el volumen aserrable por hectárea para los tres subtipos es de $7.760,2.000$ y 3.950 pulgadas madereras, respectivamente, lo que significa un volumen aserrable disponible para cada subtipo de $46.560 .000,3.000 .000$ y 2.370 .000 pulgadas madereras, que corresponden en términos porcentuales a $89.7 \%, 5.8 \%$ y $4.5 \%$, respectivamente, del volumen aserrable posible de utilizar.
$\mathrm{Si}$ suponemos que 200.000 pulgadas anuales de madera de $F$. cupressoides son el objetivo de producción del sector, se deberían extraer anualmente 179.400 pulgadas madereras (23 hectáreas) del subtipo Alerce-Coigüe de Chiloé; 11.600 pm. (5.8 hectáreas) de Alerce-Coigüe de Magallanes, y 9.000 p.m. (2.3 há) de Alerce-Ciprés. Ello significaría que se tendría toda la superficie talada en aproximadamente 460 años.

Los cálculos de crecimiento realizados (cuadro 3 ) indican, en el mejor de los casos, un crecimiento diametral anual de $1.23 \mathrm{~mm}$, y en el promedio, de $0.94 \mathrm{~mm}$, lo cual representa una edad de rotación física de más de 400 o más de 500 años, si se piensa en un diámetro de corta de $50 \mathrm{~cm}$. Este hecho descarta la posibilidad de un método silvicultural, salvo que se rebaje a la mitad la meta de producción.

Las especiales y difíciles condiciones edáficas, geomorfológicas, climáticas y vegetacionales del tipo forestal y las insospechadas consecuencias que puede tener una tala rasa sobre este medio ambiente, a lo que hay que sumar el valor paisajístico y turístico implícito en el bosque y en la región, hacen impracticable la aplicación de tala rasa en estos bosques.

El método del árbol semillero es similar desde muchos puntos de vista al de tala rasa, particular- 


\section{CUADRO 3}

Incremento promedio en diámetro y altura para distintos tipos de bosques dentro del

Tipo Forestal Alerce de Contao, Cordillera de los Andes.

Average diameter and height growth for different stand types within the

Alerce Forest Type in the Andean Cordillera of Contao.

\begin{tabular}{|lccccccc|}
\hline Sector & $\begin{array}{l}\text { DAP } \\
(\mathrm{cm})\end{array}$ & $\begin{array}{c}\text { Altura } \\
(\mathrm{m})\end{array}$ & $\begin{array}{c}\text { Edad } \\
(\text { años })\end{array}$ & $\begin{array}{c}\text { Doble espesor } \\
\text { corteza }(\mathrm{cm})\end{array}$ & $\begin{array}{c}\text { Medio anual } \\
\text { diámetro }(\mathrm{mm})\end{array}$ & $\begin{array}{c}\text { Medio anual } \\
\text { altura }(\mathrm{cm})\end{array}$ & $\begin{array}{c}\text { Periódico } 10 \text { años } \\
\text { diámetro }(\mathrm{mm})\end{array}$ \\
\hline $\begin{array}{l}\text { Patamai } \\
\text { Bajo }\end{array}$ & 149 & 40.1 & 1.169 & 8.05 & 1.23 & 3.54 & 11.89 \\
$\begin{array}{l}\text { Patamai } \\
\text { Intermedio }\end{array}$ & 60 & 19.3 & 642 & 4.9 & 0.86 & 2.95 & 8.66 \\
Volcán & 25 & 11.5 & 280 & 3.0 & 0.74 & 2.82 & 7.86 \\
Apagado & & & & & & & \\
\hline
\end{tabular}

mente en lo que se refiere a la fuerte alteración del medio y al deterioro paisajístico. Por otra parte se tiene la experiencia de que al dejar árboles o grupos de árboles aislados, la probabilidad de caída por efecto del viento es muy alta, llegando en casos al $90 \%$ en la Cordillera de la Costa (Garrido et al., 1984).

Para los subtipos Alerce-Coigüe de Magallanes y Alerce-Ciprés, cuya estructura de tamaño es del tipo de bosque multietáneo, donde la mayor parte de los árboles se encuentra en las clases de diámetro inferiores a $50 \mathrm{~cm}$ (Donoso et al., 1987; Donoso et al., en prensa), las cortas de tala rasa y árbol semillero implicarían la eliminación, sin provecho, de todos esos ejemplares, lo que, dadas las características de crecimiento del bosque, hace que ambos métodos sean más descartables aun para esos subtipos.

Métodos de cortas de protección. El método de cortas de protección clásico puede aplicarse en forma uniforme, en grupos o bosquetes, o en fajas o franjas. Estos métodos tendrían seguramente ventajas desde el punto de vista paisajístico y de protección de suelos y, probablemente, permitirían establecer con cierta facilidad la regeneración. Sin embargo, su aplicación implica abrir el dosel y aumentar los espaciamientos, lo que provoca caída de árboles, como ya fue señalado. Además, las dificultades del sustrato, en el caso del subtipo Alerce-Coigüe de Chiloé y Alerce-Ciprés de las Guaitecas, para las faenas de explotación en las cortas sucesivas o final posteriores, y el enorme tamaño de los árboles provocarían seguramente gran destrucción de la regeneración establecida. Estos métodos significan corta de árboles de ta- maños menores, que no tienen posibilidad de mercado, para dejar espacio para la regeneración y para el ingreso de luz al piso del bosque, lo que lo hace carecer de sentido, dado el alto valor de la madera y el lento crecimiento de $F$. cupressoides, particularmente en los casos de bosques multietáneos, como el de Alerce-Ciprés y Alerce-Coigüe de Magallanes. En definitiva, este método no es recomendable para la especie.

Método de cortas de selección. Queda para la discusión sólo el método de selección, el que se diferencia básicamente de todos los otros, porque, a través de su aplicación, se busca mantener o llevar el bosque a una estructura multietánea. Si uno de los objetivos principales de la silvicultura de $F$. cupressoides es el de asegurar la permanencia del recurso con todos sus valores científicos, paisajísticos y culturales, el método de selección sería el indicado. El método clásico consiste en la selección de árboles individuales que se extraen, permitiendo que la regeneración aparezca en el claro dejado. Considerando que $F$. cupressoides es una especie más bien de tolerancia media a la sombra, este método debe garantizar que los claros provean de suficiente luminosidad; ello se consigue en general porque el gran tamaño de los árboles que se extraen asegura la creación de claros grandes; si ellos no fueran suficientes, se podría aplicar la variante de selección en grupos de árboles. Este método es teóricamente posible de aplicar en el tipo forestal de la Cordillera de los Andes, pero las características topográficas y del sustrato, especialmente del subtipo Alerce-Coigüe de Chiloé, a las cuales ya se ha hecho referencia, lo hacen de dificultosa aplicación por la falta total de vías 
de acceso y la necesidad, bajo esas condiciones, de búsqueda anual de árboles que el método exige, además de su extracción del bosque.

Sin embargo, una variante del método de selección que evita los problemas y dificultades encontrados en los anteriores es el que se aplica en fajas angostas. En este método, en la medida en que se va cortando una faja y regenerando la cortada anteriormente, se va creando una secuencia Completa de clases de edad, dispuesta en el sentido del viento dominante, de modo que con el tiempo el bosque en conjunto será estructura multietánea (fig. 1).

Puesto que este método implica tala rasa en las fajas angostas, no cabe estrictamente aplicarlo en los subtipos Alerce-Coigüe de Magallanes y Alerce-Ciprés de las Guaitecas que ya poseen estructura multietánea. El método se adecúa más bien al subtipo Alerce-Coigüe de Chiloé porque implica la corta de árboles jóvenes, que en este subtipo son muy escasos. Dentro de este subtipo este método de selección parece el adecuado, porque no significa cortas masivas en corto tiempo y tampoco búsqueda de individuos y extracción posterior de ellos en medio de bosques con las características de los de este subtipo; por otra parte, protege a los alerces de caídas provocadas por el viento y facilita el volteo y madereo sin afectar a la regeneración, con la disminución correspondiente de costos.

Previamente se había señalado que la superficie apta para utilización maderera a través de aplicación de métodos silvícolas era del orden de 6.000 hectáreas para este subtipo, con un volumen de 46.560.000 pulgadas madereras de $F$. cupressoides y aproximadamente 4 millones de pulgadas madereras de otras especies, principalmente $\mathrm{No}$ thofagus nitida.

Para verificar la aplicabilidad de este método en el subtipo supongamos que la meta de produc- ción es ahora de 100.000 pulgadas madereras de F. cupressoides, que el diámetro mínimo de corta será de $50 \mathrm{~cm}$ y las fajas de $30 \mathrm{~m}$ de ancho por $200 \mathrm{~m}$ de largo (= 0.6 há). El volumen por hectárea es de 7.760 p.m., lo que entrega 4.656 p.m. por faja de 0.6 há; si se requieren 100.000 p.m. deberán cortarse 21.53 fajas, o, lo que es lo mismo, 13 hectáreas anuales mediante fajas. Si así fuere, el subtipo Alerce-Coigüe de Chiloé disponible se terminaría de cortar en 460 años, cifra que es mayor que la edad de rotación con diámetro de corta de $50 \mathrm{~cm}$ (407 años para un crecimiento diametral de $1.23 \mathrm{~mm}$ ).

Para los subtipos Alerce-Coigüe de Magallanes y Alerce-Ciprés, como se señaló, el método no cabe porque implica perder la regeneración establecida y todos los árboles menores de $50 \mathrm{~cm}$ de diámetro. Frente a esta situación es posible la aplicación de una combinación del método de selección en fajas angostas con uno de protección en las fajas. Ello significa la corta en el mismo tipo de fajas, pero sin cortar los árboles menores al diámetro límite de corta, los que quedan en pie en la faja constituyendo una regeneración adelantada y protección para la regeneración futura en la faja. Este método dejaría árboles aislados que caen con el viento; por lo tanto, es fundamental dejar alrededor de ellos grupos de árboles de otras especies que circunden y protejan a los alerces aislados. En las áreas con pendiente las fajas deberán trazarse idealmente siguiendo a las curvas de nivel, para evitar el deterioro del suelo y de la regeneración por efectos de escurrimiento.

De acuerdo con las restricciones establecidas para estos subtipos, se tienen 1.500 hectáreas susceptibles de ser intervenidas en el subtipo AlerceCoigüe de Magallanes y 600 en el subtipo AlerceCiprés de las Guaitecas. Si el diámetro límite es de $50 \mathrm{~cm}$, el volumen aserrable por hectárea es de $1.890 \mathrm{pm}$. para el primero y 3.710 para el segun-

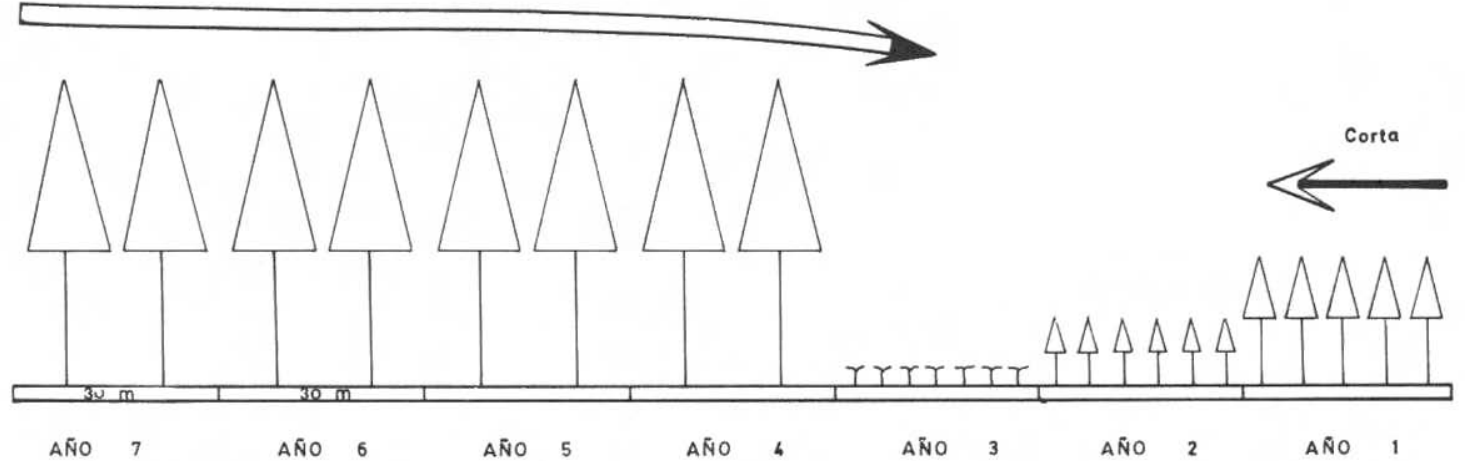

Fig. 1: Aplicación de un método de selección en fajas angostas. (Adaptado de Hawley y Smith, 1972.) Application of the selection method in narrow bands. (Adapted from Hawley and Smith, 1972.) 
do, que representan un total de 2.835.000 p.m. y 2.226 .000 p.m., respectivamente. Suponiendo una producción de 50.000 p.m. anuales para estos bosques, las que deberían extraerse proporcionalmente de ambos subtipos, se deberían extraer anualmente 28.000 p.m. del subtipo AlerceCoigüe de Magallanes y 22.000 p.m. del subtipo Alerce-Ciprés. A razón de 1.890 p.m. por há para el primero y de 3.710 para el segundo, se requerirá cortar 25 fajas de 0.6 há (15 hectáreas) y 10 fajas de 0.6 há (6 hectáreas) anuales en cada uno de ellos, respectivamente. Puesto que son 1.500 há del uno y 600 del otro, ellas se terminarán de cortar en 100 años cada una. Ese volumen adicional de 50.000 pulgadas madereras anuales por 100 años podría complementar la producción del subtipo Alerce-Coigüe de Chiloé durante los primeros años para efectos de construcción de caminos e infraestructura de explotación en general.

El crecimiento en diámetro de $F$. cupressoides en el subtipo Alerce-Coigüe de Magallanes es de $0.86 \mathrm{~mm}$ anuales y en Alerce-Ciprés de las Guaitecas se supone que puede ser parecido al de Alerce Coigüe de Chiloé (cuadro 3 ). Esto permite predecir un crecimiento aproximado de $1 \mathrm{~mm}$ diametral anual, lo que significa para un diámetro de corta de $50 \mathrm{~cm}$ un período de rotación de 500 años. Si estos subtipos se explotaran en los primeros 100 años, se tendría luego que esperar el término de las intervenciones del subtipo AlerceCoigüe de Chiloé para reiniciar las cortas de ellos.

Esta posibilidad silvicultural de intervenir los bosques de alerce de Contao deberá confrontarse, naturalmente, con la factibilidad de explotación y de costos de las faenas, lo que escapa de esta discusión.

Kimmins (1973) señala que un recurso no es necesariamente renovable por tratarse de un bosque, y que la condición de renovabilidad o no renovabilidad se encuentra más bien en el tiempo que un recurso demora en renovarse, más que en su carácter físico, químico o biológico. Así, un bosque de alerce que requiere 400 a 500 años para alcanzar su tamaño de valor comercial, no sería un recurso renovable en el sentido socioeconómico. Sin embargo, un bosque de crecimiento tan lento debe considerarse un recurso renovable si posee una superficie suficiente que permita extraer de él, anualmente, una cantidad de madera de valor tal que entregue una rentabilidad aceptable y satisfaga una cierta tasa de uso o de consumo razonable del producto. Al mismo tiempo, naturalmente, debe obtenerse una tasa de regeneración que permita la repoblación del bosque y mantenga su estructura, de modo tal que el ciclo de cortas pueda repetirse eternamente. El tiempo requerido para que los individuos de la especie alcancen su tamaño de explotación no tiene ninguna importancia bajo este concepto. Si los usos y gustos, o modas, de la sociedad cambian en el tiempo, lo que puede ocurrir en relación con cualquier rubro de producción, tampoco tiene ello mayor importancia, porque el bosque ha permanecido intacto y, más aún, mejorado durante el tiempo en que ha sido manejado.

En el análisis del caso particular del bosque de alerce de la Cordillera de los Andes de Contao deben considerarse, sin embargo, otros elementos además de la tasa de crecimiento, la regeneración, la calidad de la madera, la rentabilidad, la tasa de uso y la mantención de la estructura del bosque. En especial el subtipo Alerce-Coigüe de Chiloé, que representa el $57 \%$ de la superficie de esos bosques y el $90 \%$ del volumen aserrable, es un ecosistema que, además de la dominancia de árboles milenarios y monumentales, posee un sustrato milenario constituido por troncos muertos no descompuestos que forman una red cubierta de variada vegetación que se extiende sobre aguas semisubterráneas o esteros subsuperficiales y sobre suelos bajo los cuales se encuentran otros suelos fósiles milenarios (Donoso et al., 1987). Sin duda estos valores sobrepasan los que pueda tener la madera, y serían destruidos al intentar utilizarla, aun cuando fuese con los criterios conservacionistas de permanencia del recurso y rendimiento sostenido que son sustentados en el análisis silvicultural recién discutido.

A pesar de lo anterior, puede ser recomendable efectuar intervenciones periódicas en el subtipo Alerce-Coigüe de Chiloé, cuidadosamente efectuadas, con el objeto de favorecer el establecimiento de la regeneración, mediante la ampliación o creación de claros en el bosque, y de ese modo mantener su estructura poblacional cuando ella fuera necesario.

Las características de este bosque y los elementos de discusión planteados en los últimos párrafos indican que el ecosistema debe constituir parte del Sistema Nacional de Areas Protegidas, de responsabilidad del Estado. Aún más, ajuicio de los autores, este ecosistema, probablemente en conjunto con los Parques Nacionales Alerce Andino y Hornopirén, debería constituirse en una Reserva de la Biósfera.

Subtipo de alerce costero. Se ha señalado en trabajos anteriores que estos subtipos presentan volúmenes muy inferiores a los de los Andes, debido a explotación maderera centenaria (Donoso et al., 1987; Donoso et al., en prensa). Por otra parte, es de mucha importancia la gran cantidad de trozos y basas dispersos en toda el área, así como los árboles muertos en pie que aún poseen altos volúmenes de madera aprovechable (Donoso et al., 1987; Sandoval y Donoso, 1987). 
Métodos de corta de tala rasa y árboles semilleros. La información sobre superficies por categorías de pendiente obtenida de la medición efectuada en los planos tipológicos (cuadro 4) muestra que la mayor parte de la superficie se encuentra en pendientes muy suaves.

La superficie a considerar para efectos de posibles tratamientos silviculturales será en este caso aquella correspondiente a los subtipos alerce puro y alerce mixto. Si se descarta por razones de pendiente, bajo las condiciones de sustrato y de precipitación de estos bosques (Donoso et al., 1987; Donoso et al., en prensa), la superficie con pendientes superiores a $45 \%$ se tendría una superficie disponible de 834.7 hectáreas. De esta superficie podrían descontarse aproximadamente 500 hectáreas correspondientes a los bosques de alerce enano o muy achaparrado que se presentan en las partes muy altas. Esto llevaría la superficie a un total aproximado de 7.800 hectáreas. La superficie de alerce quemado o alterado debe considerarse para el manejo del bosque a futuro por su alto volumen aserrable (Sandoval y Donoso, 1987).

En relación con la aplicación de métodos silviculturales de tala rasa o de árboles semilleros en un sentido tradicional (Hawley y Smith, 1972), a muchas de las razones que los descartan, señaladas para el caso del alerce andino de Contao, se suman en estos subtipos las características de los suelos muy delgados, desarrollados sobre estratos de rocas metamórficas que restringen y dificultan seriamente la infiltración del agua, lo que determina que alteraciones como las provocadas por tratamientos como los señalados ocasionan deterioros de gran magnitud. Pero más que lo anterior, la estructura esencialmente multietánea de los bosques (Donoso et al., 1987; Donoso et al., en prensa), en que la mayor parte de los individuos y gran parte del volumen se encuentran concentrados en los diámetros inferiores a $50 \mathrm{~cm}$, hacen absurda la aplicación de dichos tratamientos.

Métodos de corta de protección. Experimentalmente se tiene conocimiento de que los árboles que quedan aislados después de cortas tipo raleo sufren problemas derivados de alteraciones de drenaje, que inciden seriamente en la muerte de árboles en pie o los predisponen a caídas por debilitamiento (Garrido, 1984; Donoso et al., 1987). Este hecho descarta en general la aplicación de los métodos de protección en estos subtipos.

Métodos de cortas de selección. Las características de multietaneidad de los subtipos de la Cordillera de la Costa, así como las condiciones de los factores medioambientales a que se ha hecho referencia y las superficies y volúmenes existentes, conducen también, en este caso, a la discusión sobre la aplicación de un método de selección. Efectivamente, puesto que la idea fundamental es producir madera de alerce en forma sostenida en el tiempo, en armonía con otros valores del bosque, no es razonable acudir a otro método silvilcultural que no sea el de selección, que es el único que mantiene la estructura multietánea del bosque y no altera esencialmente su medio ambiente. En esta área de la Cordillera de la Costa de Valdivia los bosques han sido explotados desde muy antiguo y, por consiguiente, existe una infraestructura caminera que, si bien es de mala calidad y mal mantenida, permite un acceso relativamente fácil dentro de un área de pendientes suaves; es tal la cantidad de huellas

\section{CUADRO 4}

Superficie de subtipos del Tipo Forestal Alerce de la Cordillera de la Costa (Venecia), según categorías de pendiente

Area (ha) per slope classes of the subtypes of the Alerce Forest Type of the Coastal

Cordillera (Venecia).

\begin{tabular}{|c|c|c|c|c|c|c|}
\hline \multirow{2}{*}{ Subtipo forestal } & \multicolumn{4}{|c|}{ Categorías de pendiente } & \multirow{2}{*}{ Total } & \multirow{2}{*}{$\%$} \\
\hline & $0-30 \%$ & $31-45 \%$ & $46-60 \%$ & $60 \%$ y más & & \\
\hline Alerce puro & 2.444 & 230 & 198 & 107 & 2.982 & 19.5 \\
\hline Alerce mixto & 5.184 & 486 & 203 & 323 & 6.196 & 40.5 \\
\hline $\begin{array}{l}\text { Alerce alterado o } \\
\text { quemado }\end{array}$ & 5.667 & 163 & 237 & 65 & 6.132 & 40.0 \\
\hline Totales & 13.298 há & 879 há & 638 há & 495 há & 15.310há & 100 \\
\hline & $86.61 \%$ & $5.81 \%$ & $4.31 \%$ & $3.31 \%$ & $100 \%$ & \\
\hline
\end{tabular}


de madereo y vías de saca que existen en los rodales, que un ordenamiento de ellos, a través de la aplicación de un método de selección, contribuirá consistentemente a recuperar superficies para el bosque donde es fácil promover la regeneración o plantar. Los factores medioambientales, particularmente aquellos relacionados con la profundidad del suelo, la precipitación y el drenaje que restringen fuertemente la aplicación de los métodos de tala rasa, árboles semilleros y protección, no se verían afectados seriamente con la aplicación de un método de selección de árboles individuales o en grupos.

Es básico para definir si el método es factible de ser aplicado, conocer los volúmenes de que se dispone por unidad de superficie (cuadro 5). El cuadro 5 permite verificar que para pendientes de 0 a $45 \%$ en los dos subtipos, en conjunto, se dispone de un volumen aserrable de 13.303.917.5 pulgadas madereras. Si se descuenta el volumen de alerce enano en una superficie de unas 500 hectáreas, ello correspondería a 796.899.4 pulgadas madereras que, dado el tamaño de los árboles, debería reducirse a menos de la mitad. Se podría llevar el volumen disponible a una cifra de 13 millones de pulgadas madereras, equivalentes a 1.667 pulgadas madereras por hectárea.

Se debería tomar como base, entonces, una superficie total disponible de 7.800 hectáreas. El crecimiento calculado para este subtipo da valores de 1.33 a $1.52 \mathrm{~mm}$ diametrales anuales y 5.95 y $7.38 \mathrm{~mm}$ de altura anuales (cuadro 6), lo que es bastante más alto que lo encontrado en la Cordillera de los Andes. Estos valores significan que para un diámetro de $60 \mathrm{~cm}$, la edad de rotación sería de 450 a 395 años (promedio 430 años), bastante inferior a las edades estimadas para el sector andino. Considerando el valor medio de $1.4 \mathrm{~mm}$ de crecimiento medio anual en diámetro, se calculó una tasa de crecimiento volumétrico medio anual de $2.8 \mathrm{~m}^{3} / \mathrm{há}$ para el alerce costero. Considerando las características de rodalización natural del área (fig. 2) se puede establecer un tamaño de rodal de 600 há aproximadamente, lo que hace proponer un ciclo de corta de 13 años (7.800: $600=13$ ), que se puede reducir a 10, como margen de seguridad.

De este modo, si la información del inventario fuera aplicable a toda la superficie, cada año se podría talar un volumen correspondiente a 2.8 $\left(\mathrm{m}^{3} /\right.$ há) x 13 (años del ciclo de corta) x 600 (tamaño de cada rodal), es decir, $21.840 \mathrm{~m}^{3}$ de madera de alerce, que significan más de 250.000 pulgadas madereras anuales. A esto debe agregarse la madera muerta en pie o volteada, que suma más de un millón de pulgadas, que servirían para paliar los costos de mejoramiento de la infraestructura durante los primeros años.

Por otra parte, es factible aprovechar más de un millón de pulgadas de madera de otras especies, en especial $N$. nitida, Drimys winteri, Weinmannia trichosperma, Saxegothaea conspicua y Podocarpus nubigena, sin considerar a otras que se presentan en forma marginal en el tipo.

\section{CUADRO 5}

Volúmenes de Fitzroya cupressoides de los subtipos del Tipo Forestal Alerce Costero en las diferentes categorías de pendientes en el sector de Venecia.

Fitzroya cupressoides timber volumes of the subtypes of the Andean Alerce Forest Type in the different slope classes in Venecia.

\begin{tabular}{|c|c|c|c|c|c|}
\hline \multirow{3}{*}{ Subtipo forestal } & \multicolumn{4}{|c|}{ Volumen neto $\mathrm{m}^{3}$ s.s.c. especie alerce } & \multirow{3}{*}{ Total } \\
\hline & \multicolumn{4}{|c|}{ Categorías de pendientes } & \\
\hline & $0-30 \%$ & $31-45 \%$ & $46-60 \%$ & $60 \%$ y más & \\
\hline Alerce puro & 551.975 .5 & 51.888 .0 & 44.781 .6 & 24.116 .5 & 672.761 .7 \\
\hline Alerce mixto & 1.099 .981 .1 & 103.320 .2 & 42.991 .7 & 68.498 .2 & 1.314 .791 .2 \\
\hline Alerce alterado & 35.226 .6 & 3.117 .4 & 1.121 .1 & 649.2 & 40.114 .3 \\
\hline Total & 1.687 .183 .2 & 158.325 .6 & 88.894 .4 & 93.264 .0 & 2.027 .667 .2 \\
\hline \multicolumn{6}{|c|}{ Volumen pulgadas madereras especie alerce } \\
\hline Alerce puro & 2.390 .670 .6 & 224.733 .0 & 193.954 .4 & 104.451 .9 & 2.913.809.9 \\
\hline Alerce mixto & 9.770 .756 .1 & 917.757 .8 & 381.880 .8 & 608.445 .7 & 11.678 .840 .4 \\
\hline Alerce alterado & 251.266 .8 & 22.236 .0 & 7.996 .8 & 4.630 .8 & 286.130 .0 \\
\hline Total & 12.412 .693 .5 & 1.164 .726 .8 & 583.832 .0 & 717.528 .4 & 14.878 .780 .3 \\
\hline
\end{tabular}


C. DONOSO, V. SANDOVAL, R. GREZ

\section{CUADRO 6}

Incrementos promedio en diámetro y altura para distintos tipos de bosques dentro del Tipo Forestal Alerce de Venecia, Cordillera de la Costa.

Average diameter and height growth for different stand types within the Alerce Forest Type in the Coastal Cordillera of Venecia.

\begin{tabular}{|lcccccc|}
\hline Sector & DAP & Altura & $\begin{array}{c}\text { Edad } \\
(\mathrm{cm})\end{array}$ & $\begin{array}{c}\text { Media anual } \\
\text { (años) }\end{array}$ & $\begin{array}{c}\text { Incrementos } \\
\text { Media anual } \\
\text { altura }(\mathrm{cm})\end{array}$ & $\begin{array}{c}\text { Periódico 10 años } \\
\text { diámetro (mm) }\end{array}$ \\
\hline Chaihuín & 35 & 16.5 & 233 & 1.40 & 7.01 & 13.84 \\
El Encanto & 35 & 16.5 & 232 & 1.33 & 6.95 & 13.46 \\
El Olvido Colún & 41 & 18.0 & 246 & 1.52 & 7.38 & 15.20 \\
\hline
\end{tabular}

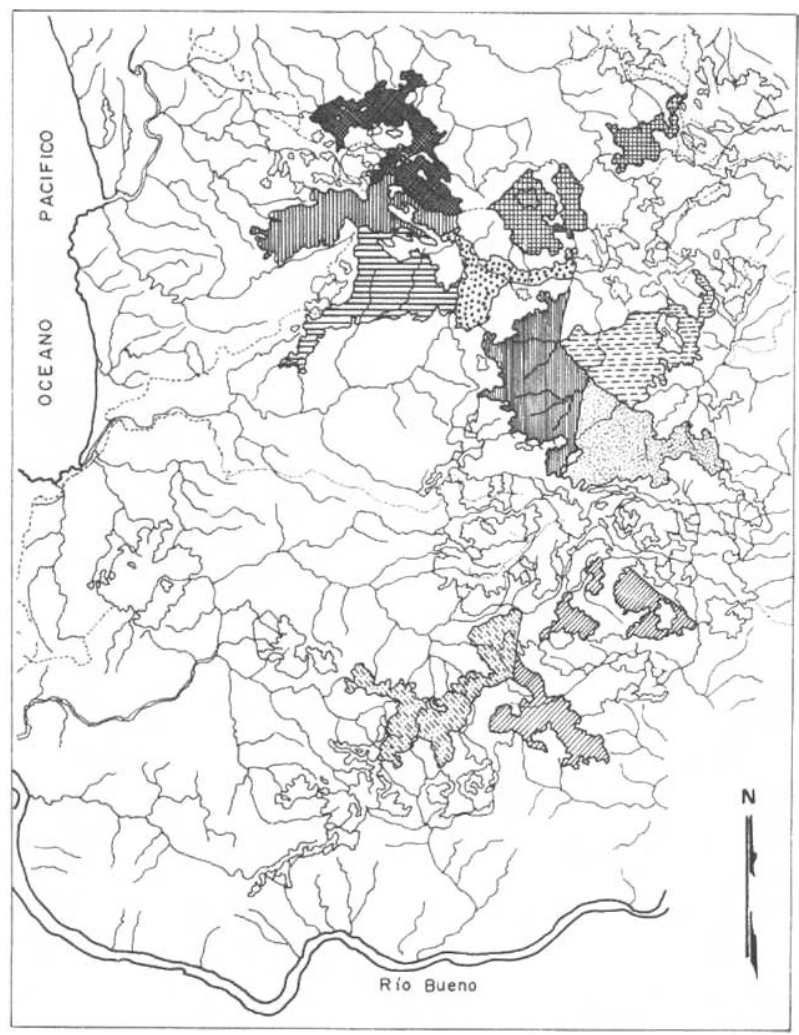

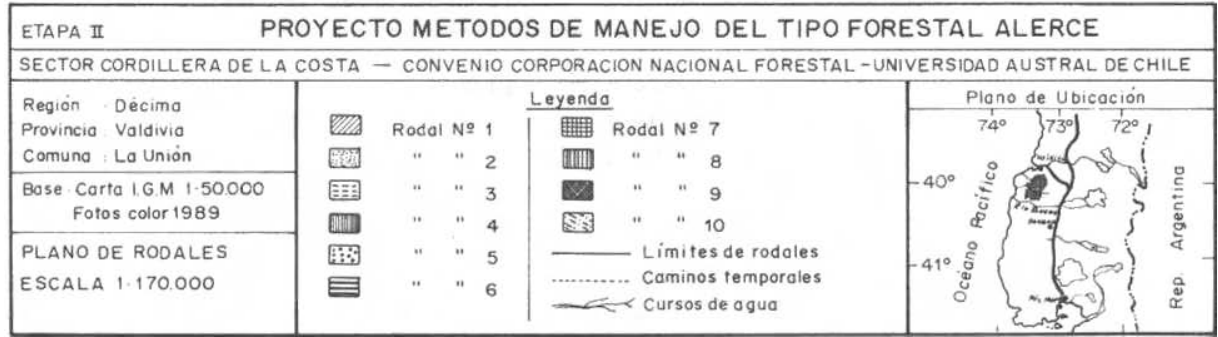

Fig. 2: Rodalización del bosque de alerce de la Cordillera de la Costa de Valdivia, considerando 10 rodales de aproximadamente 600 há cada uno para efectos de su manejo mediante el método de selección uniforme.

Stand division of the Alerce forest of the Coastal Cordillera of Valdivia, into 10 stands of around 600 ha each one, in order to be managed through the uniform selection method. 
A la luz de esta información y de la de otros trabajos (Parker y Donoso*), parece no caber duda de que en la Cordillera de la Costa el aprovechamiento y silvicultura de los bosques de alerce es una realidad más que una ficción. Su aplicación masiva y controlada en el área, según la prescripción general que aquí se entrega, permitiría ordenar adecuadamente estos bosques en un sentido silvicultural, desarrollar el área con un tipo de actividad forestal que podría ser un modelo, y regenerar o manejar la regeneración de 6.000 hectáreas alteradas, que podrían brindar belleza y bienes a las generaciones futuras, a las cuales no tenemos ningún derecho de privarlas de los beneficios de usar esta especie extraordinaria. Las características y estado actual de los bosques de alerce de la Cordillera de la Costa, entonces, hacen de la acción silvicultural en ellos, con un objetivo múltiple, no sólo una posibilidad, sino que una necesidad para acelerar el embellecimiento del paisaje a través de un método que armoniza la producción de madera con la mantención e incremento de los valores de vida silvestre, recursos hídricos y recreación. Esta situación es muy distinta a la de los bosques de Contao.

La proposición que vale aquí es la realización de un proyecto de 10 años para aplicar el método propuesto y evaluar sus resultados económicos y los efectos positivos y negativos del impacto ambiental.

\section{AGRADECIMIENTOS}

Este trabajo ha sido realizado gracias al aporte financiero de los Convenios UACH/CONAF (4200-6-53) y UACH/Forestal Venecia (4200-630) y al esforzado trabajo de terreno y gabinete de numerosos colaboradores que han funcionado adscritos a dichos proyectos.

\footnotetext{
* Comunicación personal.
}

\section{BIBLIOGRAFIA}

DANIEL, T.; HELMS, J. y BAKER, F. 1982. Principios de Silvicultura. McGraw-Hill Co. Inc., México, $492 \mathrm{pp}$.

DONOSO, C; GREZ, R.; SANDOVAL, V.; JUACIDA, R.; GAYOSO, R. y NUÑEZ, P. 1987. Zonificación, caracterización y subtipificación del Tipo Forestal Alerce. Proyecto de Manejo del Tipo Forestal Alerce. Informe de Convenio $\mathrm{N}^{\circ} 115$. Serie Técnica, Fac. Cs. Forestales, Universidad Austral de Chile.

DONOSO, C. (En prensa). Producción de semillas y hojarasca de las especies del Tipo Forestal Alerce de la Cordillera de la Costa de Valdivia. Revista Chilena de Historia Natural.

DONOSO, C; GREZ, R. y SANDOVAL, V. (En prensa). "Caracterización del Tipo Forestal Alerce", Bosque.

ELIZALDE, R. 1970. La sobrevivencia de Chile. Min. de Agricultura, Servicio Agrícola y Ganadero, Santiago.

GARRIDO, F.; URZUA, A. y BLANCO, S. 1983. Catastro del tipo Alerce. Informe Escuela de Ingeniería Forestal, Universidad Austral de Chile, $35 \mathrm{pp}$.

GARRIDO, F. 1984. "Las cortas de seminación y el peligro del viento en rodales de alerce", Ciencias Forestales. 4(1): 29-40.

HAWLEY, R. y SMITH, B. 1972. Silvicultura Práctica. Editorial Omega, Barcelona, 544 pp.

KIMMINS, P.P. 1973. "The renewability of natural resources", Journal of Forestry 71: 5.

KUHNE, A. 1985. Estudio pedológico y geomorfológico de Contao a Río Negro en la X Región de Los Lagos. Boletín Técnico 20, CONAF, 41 pp.

PEREZ ROSALES, V. 1850. Recuerdos del pasado. Editorial Pomaire, Santiago.

SANDOVAL, V. y DONOSO, C. 1987. Informe Técnico sobre Inventario de alerce muerto en bosque quemado del predio Venecia en la Cordillera de la Costa. Informe de Convenio $\mathrm{N}^{\circ}$ 126. Serie Técnica, Fac. Cs. Forestales, Universidad Austral de Chile.

VEBLEN, T.T.; DELMASTRO, R. y SCHLATTER, J. 1976. "The conservation of $F$. cupressoides and its environment in Southern Chile", Environmental Conservation. 3: 291-301.

VEBLEN, T.T. y ASHTON, D.A. 1982. "The regeneration status of $F$. cupressoides in the Cordillera Pelada. Chile", Biological Conservation 23: 141161 . 